\title{
Photonic Molecules in Photonic Crystals
}

\author{
Satoru Ishii, Kengo Nozaki and Toshihiko Baba \\ Department of Electrical and Computer Engineering, Yokohama National University \\ 79-5 Tokiwadai, Hodogaya-ku, Yokohama 240-8501, Japan \\ E-mail address baba@ynu.ac.jp
}

\begin{abstract}
We propose a photonic molecule consisting of multiple nanocavities in a photonic crystal and demonstrate its lasing and modal characteristics by finite-difference time-domain analysis and by experiments. In the analysis, we show that a point-shift defect and a point-missing defect have s- and p-orbital cavity modes, respectively, and that two adjacent defects exhibit $\sigma$ - and $\pi$-orbital bonding and antibonding modes, which are similar to electronic states in chemical molecules. For such structure, we verify the modal characteristics as photonic molecules, i.e., mode splitting and its dependence on coupling strength, through their fabrication into GaInAsP photonic crystal slabs and the observation of lasing characteristics.
\end{abstract}

KEYWORDS: photonic molecule, photonic crystal, nanolaser, coupled cavity laser, GaInAsP

\section{Introduction}

Photonic molecules (PMs) are optical analogs to chemical molecules, i.e., coupled cavities whose resonant modes are similar to electronic states in chemical molecules. Such similarities have been discussed for PMs consisting of coupled two-vertical-cavity surface-emitting lasers ${ }^{1)}$ and for coupled microspheres. ${ }^{2)}$ In a recent study, we fabricated PMs of two or more microdisk lasers (MDLs) and observed their photopumped lasing as well as their anti-crossing modal characteristics and mini-band formation arising from mode coupling. ${ }^{3)}$ We also observed a bistability based on saturable absorption or gain switching in the MDL-PM by separating gain and absorption regions. ${ }^{4,5)}$ The threshold of the bistability is less than $100 \mu \mathrm{W}$ even under continuous wave operation at room temperature. Thus, MDLs are effective for demonstrating the fundamental properties and useful functions of PMs. However, one disadvantage of the MDL-PM is higher order mode coupling, which is less similar to simple chemical molecules such as $\mathrm{H}_{2}$. The MDL has a whispering gallery mode (WGM) as a cavity mode, which maintains a high $Q$ because of total internal reflection at the disk edge. This mode cannot be the fundamental mode or a lower order mode but a higher order mode beyond the 10th, and its volume is typically as large as $10\left(\lambda / n_{1}\right)^{3}$, where $\lambda$ is the resonant wavelength and $n_{1}$ is the index of the active layer. ${ }^{6,7)}$

In this study, we propose and demonstrate PMs in a photonic crystal (PC). A point defect introduced into a uniform PC can be a nanocavity laser. ${ }^{8-11)}$ It maintains a high $Q$ fundamental or lower order mode with a volume of less than $\left(\lambda / n_{1}\right)^{3}$. Therefore, two or more adjacent point defects are a PM exhibiting greater similarities to simple chemical molecules. In the MDL-PM, the coupling is very sensitive to the interdisk spacing, because it takes place between exponentially decaying evanescent fields of modes. On the other hand, coupling is easier to control in the PC-PM, because the mode is confined by Bragg reflection, which has a greater penetration depth than the total internal reflection.

In this study, we present finite difference time domain (FDTD) analysis of PC-PMs in $\S 2$. Here, we first show that two kinds of original cavity modes correspond to s- and p-orbital electrons. Then we discuss the bonding and antibonding of the original modes in PC-PMs, which are similar to $\sigma$ - and $\pi$-orbital electrons in chemical molecules. In $\S 3$, we describe the fabrication of the PC-PMs into GaInAsP PC slabs and the observation of photopumped lasing. We also evaluate mode coupling from the splitting of the resonance and its dependence on the coupling strength.

\section{Theoretical Analysis}

In the analysis, a PC slab consisting of circular airholes arranged in a triangular lattice is assumed as the fundamental model, and the modal characteristics of defects and PMs are calculated 
using a two-dimensional FDTD method with the equivalent index approximation of the slab. The normalized airhole diameter $2 r / a$ is set to 0.545 for the lattice constant $a$, the equivalent index of the slab $n_{\mathrm{eq}}=2.73$, the index of airholes is 1.0 , and the Yee cell size is $a / 31.4$. The initial wave is given for the vertical component of the magnetic field $H_{\mathrm{z}}$ by a Gaussian pulse with a center-normalized frequency $a / \lambda$ of 0.275 . This induces transverse electric polarization, for which the PC slab has an in-plane photonic bandgap.

Figure 1 shows the distributions of $H_{\mathrm{z}}$ and the in-plane electric field vector $\boldsymbol{E}_{\mathrm{xy}}$ of original cavity modes for two kinds of point defects with the coordinate system and corresponding Brillouin zone. Figure 1(a) assumes a point-shift defect, in which two adjacent airholes are each shifted by $0.17 a{ }^{11)}$ In the following, we refer to this as an $\mathrm{H} 0$ defect. The figure shows a monopole $H_{\mathrm{z}}$ distribution with no nodes in the cavity. Due to the differential relations $E_{\mathrm{x}} \propto \partial H_{\mathrm{z}} / \partial y$ and $E_{\mathrm{x}} \propto-\partial H_{\mathrm{z}} / \partial x, \boldsymbol{E}_{\mathrm{xy}}$ rotates around the $H_{\mathrm{z}}$ pole. Because it looks like an s-orbital electron in an atom, we refer to it as an s mode. Figure 1(b) assumes a point-missing defect, usually called an $\mathrm{H} 1$ defect, in which one airhole is removed. This structure should maintain three-fold rotationally symmetric modes having dipole $H_{\mathrm{z}}$ distributions with one node and figure-of-eight $\boldsymbol{E}_{\mathrm{xy}}$ distributions. It is well known, however, that the degeneracies in one direction ( $x$ direction in the case of Fig. 1 ) and in the two other directions are broken, because the symmetry of the actual calculation model (and fabricated devices) is not perfect. As a result, two dipole modes in the $x$ and $y$ directions appear with different resonant frequencies. They act as different original modes in the PMs, as shown below. Since they look like p-orbital electrons in an atom, we call them $\mathrm{p}_{x}$ and $\mathrm{p}_{y}$ modes. Thus, the original modes in the PC are very simple, compared with whispering gallery modes in the MDL.

Figure 2 shows the field distributions in a PM consisting of two $\mathrm{HO}$ defects. Here, the defects are aligned on the $y$ axis $(\Gamma-\mathrm{X} \mathrm{PM})$, and the number of airholes $N$ separating the defects is set to 1 . In general, two modes characterized by in-phase and out-of-phase coupling appear in diatomic PMs. Note here that the coupling phase in $H_{\mathrm{z}}$ is opposite that in $\boldsymbol{E}_{\mathrm{xy}}$ due to the differential relationships. In most cases, $\boldsymbol{E}_{\mathrm{xy}}$ has a higher intensity than $H_{\mathrm{z}}$ on or near the centerline between the two defects because of the discontinuity in $\boldsymbol{E}_{\mathrm{xy}}$ at the boundary of airholes separating the defects. This means that $\boldsymbol{E}_{\mathrm{xy}}$ (particularly the electric field component parallel to the centerline, which contributes to the coupling) mainly determines the parity of the coupling. Therefore, we define the bonding and antibonding modes as those showing in-phase and out-of-phase couplings of $\boldsymbol{E}_{\mathrm{xy}}$, respectively. The distribution in Fig. 2 also shows a similarity to the situation in which s-orbital electrons form $\sigma$-orbital bonding and antibonding states in a chemical molecule. Therefore, we call this $\sigma$-coupling. Figure 3 shows the field distributions in PMs consisting of the two H1 defects shown in Fig. 1(b). As shown in Fig. 3(a), the PM with two defects aligned on the $x$ axis ( $\Gamma-\mathrm{J}$ PM) exhibits bonding and antibonding modes formed by the coupling of two $p_{x}$ or $p_{y}$ modes shown in Fig. 1(b) (four modes in total). In the bonding and antibonding modes, the two $\mathrm{p}_{x}$ modes exhibit $\sigma$-coupling, whereas the two $\mathrm{p}_{\mathrm{y}}$ modes exhibit $\pi$-coupling, which is similar to the situation in which p-orbital electrons form $\pi$-orbital states in a chemical molecule. Four similarly coupled modes can be seen for the $\Gamma$-X PM with two H1 defects, as shown in Fig. 3(b). Thus, PC PMs provide a better analogy to chemical molecules than MDL PMs.

The formation of bonding and antibonding modes leads to a resonant frequency splitting, the width of which depends on the coupling strength. Figure 4 shows the normalized frequency $a / \lambda$ of each mode in the PC-PMs with H1 defects calculated as a function of $N$. The splitting becomes wider as $N$ decreases. In addition, it is wider for $\sigma$-coupling and in the $\Gamma$-X PM than for $\pi$-coupling and in the $\Gamma$-J PM. In particular, the splitting is wide in the $\Gamma$-X PM even for $N=5$ and a corresponding interdefect spacing of $2 \sqrt{3} a+2 r(\sim 1.8 \mu \mathrm{m}$ in the experiment shown in the next section). Thus, in PC PMs, the mode coupling and splitting are obtained more easily than in MDL PMs, in which the interdisk spacing has to be narrower than $0.2 \mu \mathrm{m} .{ }^{3)}$ The difference in the splitting between modes and structures arises from the different field penetration depths of the original mode; the $\mathrm{p}_{\mathrm{y}}$ mode penetrates more than the $\mathrm{p}_{\mathrm{x}}$ mode, and the $\mathrm{p}_{\mathrm{y}}$ mode penetrates more in the $y$ direction than in the $x$ direction. Since the $\pi$-coupling is particularly weak, the splitting cannot be observed for $N>1$ in the analysis. In Fig. 4, circles and triangles denote the bonding and antibonding modes, respectively. In most cases, the bonding mode frequency is lower than the antibonding mode frequency. This supports our definition of bonding and antibonding modes. One exceptional case is the $\pi$-coupling of the $\Gamma$-J 
PM. Frequencies of the bonding and antibonding modes are close, but the former is slightly higher than the latter. Such an opposite situation may occur due to the very weak intensity of $\boldsymbol{E}_{\mathrm{xy}}$ in the PM near the centerline between the defects. In this case, $H_{\mathrm{z}}$ mainly determines the parity of the coupling, and thus, the frequency relation between the bonding and antibonding modes is inverted (or the definition of these modes should be changed, although we keep the initial definition in the following discussion to avoid confusion).

\section{Experiment}

In device fabrication, we used GaInAsP/InP epitaxial wafers. The active layer in each wafer consisted of five compressively strained quantum wells, and its total thickness and photoluminescence peak were 0.2 and $1.58 \mu \mathrm{m}$, respectively. For this wafer, an airbridge PC slab in a triangular lattice was formed using $e$-beam lithography, $\mathrm{HI} / \mathrm{Xe}$ inductively coupled plasma etching, ${ }^{12)}$ and $\mathrm{HCl}$ wet etching of InP. Scanning electron microscopy (SEM) images of fabricated PC-PMs with $\mathrm{H} 0$ defects and $\mathrm{H} 1$ defects are shown in Fig. 5. Here, the lattice constant $a$ is 0.46 and $0.44 \mu \mathrm{m}$ for the former and latter, respectively, and the basic airhole diameter $2 r$ is $0.22 \mu \mathrm{m}$ ( $2 r / a=0.50$ for the latter). The exceptional airhole diameter $2 r^{\prime}$ around the defects is set to be $0.20 \mu \mathrm{m}\left(2 r^{\prime} / a=0.45\right.$ for the latter) to reduce out-of-plane radiation loss. ${ }^{10)}$ The number of airholes $N$ is changed between samples.

In the measurement, the entire area of the PM was photopumped by $0.98 \mu \mathrm{m}$ pulsed laser light at room temperature at a duty ratio of $0.075 \%$ and a focused spot diameter of $3.5 \mu \mathrm{m}$. Output light from the device was coupled to an optical fiber through the upper lens system after filtering the pump light and analyzed using an optical spectrum analyzer. The lasing characteristics measured for the $\Gamma-\mathrm{X} P M$ of H0 defects with $N=1$ and the $\Gamma-\mathrm{J}$ PM of H1 defects with $N=2$ are shown in Fig. 6. Two neighboring mode peaks were observed for both devices. Regarding the PM with H0 defects, only this device lased. Therefore, it is difficult to discuss modal behavior as a PM for this sample. On the other hand, we observed lasing in many samples of PMs with H1 defects. The typical threshold pump power was $1.8-2.0 \mathrm{~mW}$. This threshold value is nearly twice that for a single H1 defect laser fabricated by the same process. ${ }^{12)}$ This result is reasonable because the optical gain was divided into the two modes. When $2 r$ was reduced to $0.20 \mu \mathrm{m}(2 r / a=0.46), 2 r$ ' was also reduced due to the proximity effect of $e$-beam lithography, and the lasing wavelengths became $10-30 \mathrm{~nm}$ longer. This should mainly be caused by the change in the cavity length. For a $10 \mathrm{~nm}$ reduction in $2 r$ ', the cavity length measured from the space between the innermost airholes becomes $\sim 1.5 \%$ smaller. It corresponds to a wavelength shift of $\sim 20 \mathrm{~nm}$ at a lasing wavelength $\lambda \sim 1.6 \mu \mathrm{m}$.

Figure 7 shows the dependence of two lasing wavelengths on $N$. For the $\Gamma-\mathrm{J}$ PM, wavelength splitting was clearly observed for $N<5$, and it was increased by decreasing $N$, as shown in Fig. 7(a). This result indicates that two-mode lasing arises from the bonding and antibonding modes. It is difficult to directly specify whether the lasing occurred by $\sigma$-coupling or $\pi$-coupling. Therefore, we carried out three-dimensional (3D) FDTD simulation by precisely modeling the experimental device. We found that the observed modes are the bonding and antibonding modes of the $\pi$-coupling, because the out-of-plane loss of the $\sigma$-coupling is very large, and only the $\pi$-coupling shows $Q$ factors higher than 1000 for $N<2$. The splitting in Fig. 7(a) is much wider than the calculated one shown in Fig. 4(a). This may be caused by the size difference between the two defects. As mentioned above, a 10 $\mathrm{nm}$ change in $2 r$ ' shifts the lasing wavelength by $\sim 20 \mathrm{~nm}$ and changes the mode-splitting characteristics. Although Fig. 7(a) suggests that the splitting arises partly from the coupling as it decreased with larger $N$. For the $\Gamma$-X PM, two-mode lasing was not confirmed but only single-mode lasing, as shown in Fig. 7(b). The 3D FDTD calculation also indicated that only antibonding modes of the $\sigma$-coupling and the $\pi$-coupling have high $Q$ s and in particular, the former has a high $Q$ of 3500 . Therefore, single-mode lasing is a reasonable result for this PM.

\section{Conclusions}

We proposed PMs formed by point defect nanocavities in the PC slab. First, we theoretically showed that the $\mathrm{H} 0$ defect and the $\mathrm{H} 1$ defect have s-orbital and p-orbital resonant modes, respectively, as original modes, and that PMs formed by these defects exhibit $\sigma$-orbital and $\pi$-orbital bonding and antibonding modes. Such simple original modes and mode couplings analogous to those in chemical 
molecules and easy couplings due to the large field penetration from the cavity are unique features of PC PMs which cannot be obtained in MDL PMs. We fabricated PMs with H0 and H1 defects in GaInAsP slabs, and observed two-mode lasing by photopumping. The dependence of the mode splitting on the coupling strength was suggested for the H1 PM. In PCs, various PMs can be formed by lithography which will be an interesting area for future study. Another future target is the fabrication of a photonic integrated circuit, in which functional PM devices such as bistable switches are monolithically integrated with other PC devices.

\section{Acknowledgment}

This work was supported in part by a Grant-in-Aid, the IT Program, and the 21st Century COE Program of Ministry of Education, Culture, Sports, Science and Technology, and a Grant-in-Aid from Japan Society for the Promotion of Science.

\section{References}

1) M. Bayer, T. Gutbrod, J. P. Reithmaier and A. Forchel: Phys. Rev. Lett. 81 (1998) 2582.

2) T. Mukaiyama, K. Takeda, H. Miyazaki, Y. Jimba and M. Kuwata-Gonokami: Phys. Rev. Lett. 82 (1999) 4623.

3) A. Nakagawa, S. Ishii and T. Baba: Appl. Phys. Lett. 86 (2005) 041112.

4) S. Ishii and T. Baba: Appl. Phys. Lett. 87 (2005) 181102.

5) S. Ishii, A. Nakagawa and T. Baba: IEEE J. Sel. Top. Quantum Electron. 12 (2006) 71.

6) T. Baba: IEEE Sel. Top. Quantum Electron. 3 (1997) 808.

7) M. Fujita, R. Ushigome and T. Baba: IEEE Photonics Technol. Lett. 13 (2001) 403.

8) M. Loncâr, M. Hochberg, A. Scherer and Y. Qiu: Opt. Lett. 29 (2004) 721.

9) H.-Y. Ryu, M. Notomi, E. Kuramochi and T. Segawa: Appl. Phys. Lett. 84 (2004) 1067.

10) T. Baba, D. Sano, K. Nozaki, K. Inoshita, Y. Kuroki and F. Koyama: Appl. Phys. Lett. 85 (2004) 3989.

11) K. Nozaki, T. Ide, J. Hashimoto, W-H. Zheng and T. Baba: Electron. Lett. 41 (2005) 843.

12) T. Ide, J. Hashimoto, K. Nozaki, E. Mizuta and T. Baba: Jpn. J. Appl. Phys. 45 (2006) L102.
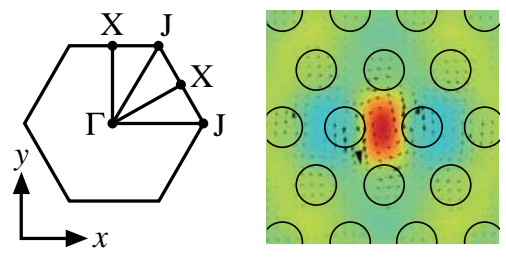

(a)

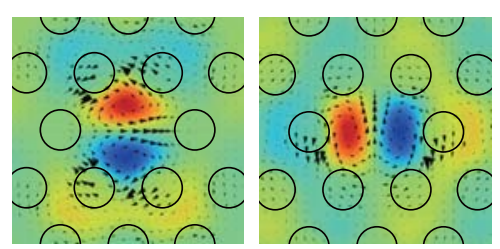

(b)

Fig. 1 FDTD model and field distributions of original modes with coordinate system and Brillouin zone. (a) $\mathrm{H} 0$ defect. (b) $\mathrm{H} 1$ defect. Colors show $H_{\mathrm{z}}$ field with relative intensity from -1 (blue), 0 (green) to 1 (red). Arrows denote $\boldsymbol{E}_{\mathrm{xy}}$ vectors.

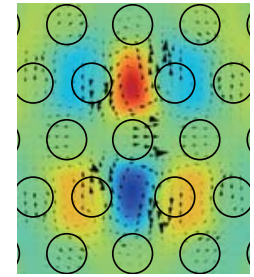

(a)

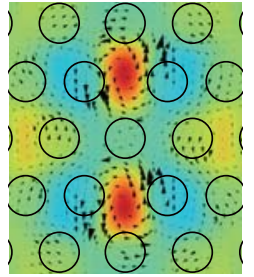

(b)
Fig. 2 FDTD models of PMs consisting of H0 defects and field distributions of modes. (a) Bonding mode. (b) Antibonding mode. 


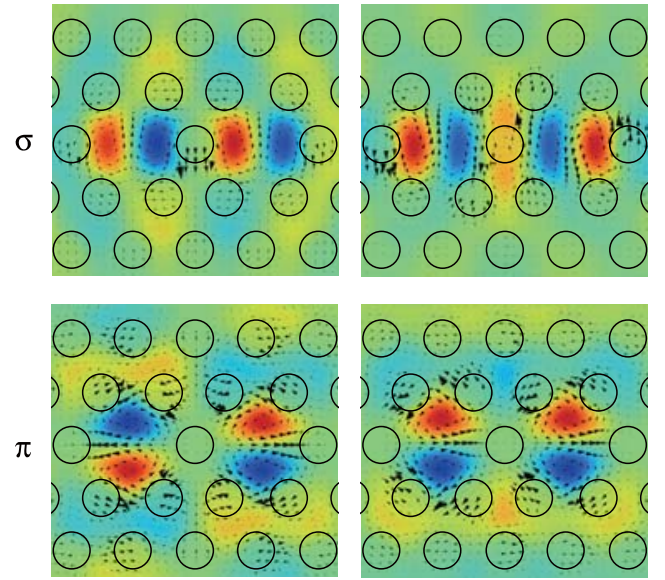

(a)
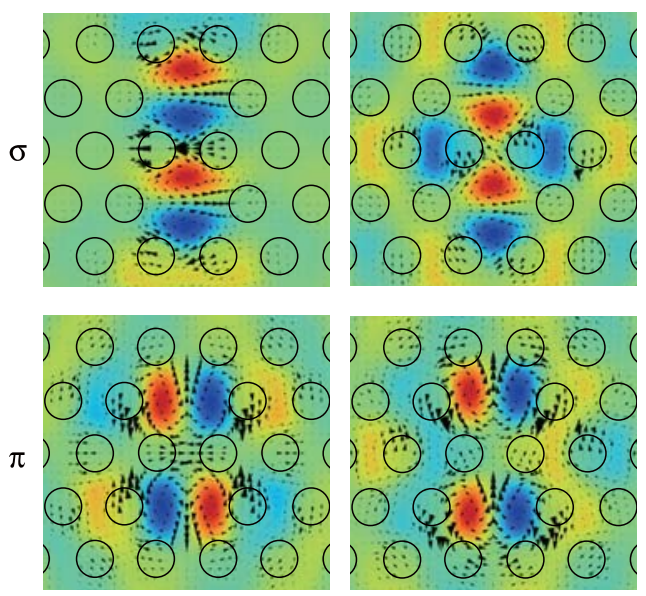

(b)

Fig. 3 FDTD model of PMs consisting of H1 defects and field distributions of modes. (a) $\Gamma$-J PM. (b) $\Gamma$-X PM. Images on the left shows the bonding mode, and those on the right, the antibonding mode.

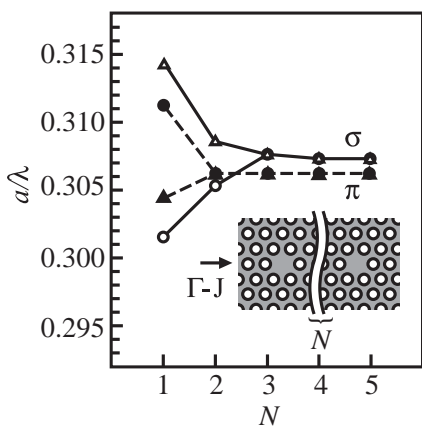

(a)

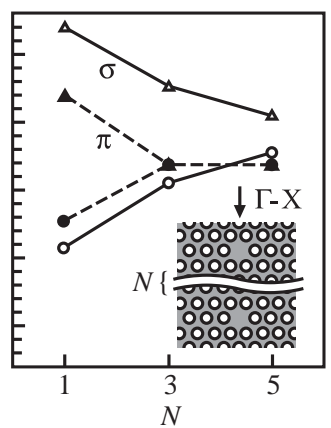

(b)
Fig. 4 Calculated normalized frequency $a / \lambda$ of coupled modes, when $N$ is the number of airholes separating defects. (a) $\Gamma-J$ PM. (b) $\Gamma$-X PM.
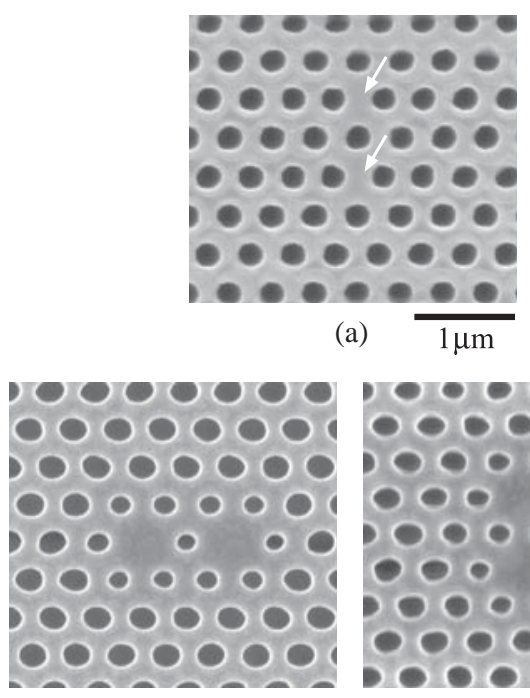

(b)

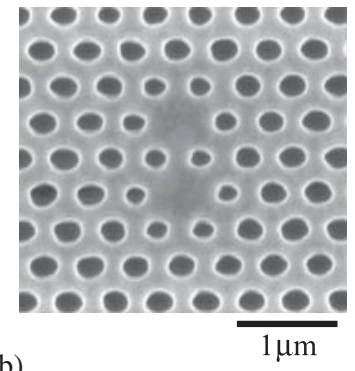

Fig. 5 Top views of fabricated devices. (a) PM with $\mathrm{H} 0$ defects. Two white arrows indicate positions of H0 defects. (b) PMs with H1 defects. The left image shows $\Gamma-\mathrm{J}$ PM, and the right $\Gamma$-X PM.

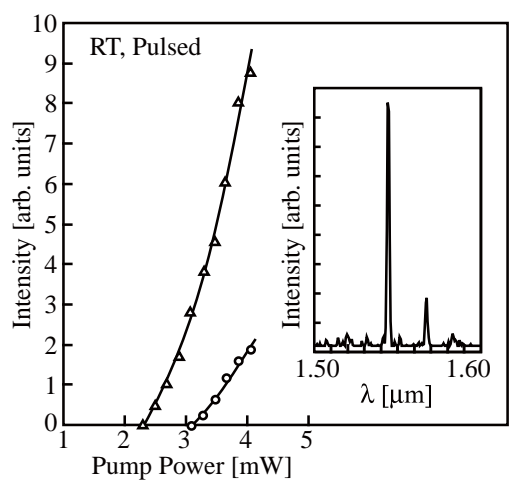

(a)

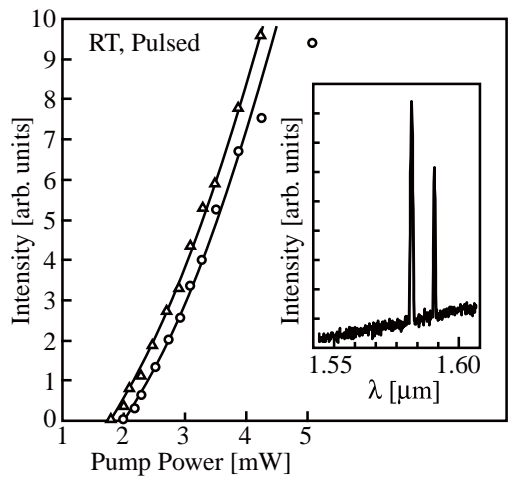

(b)

Fig. 6 Lasing characteristics. (a) PM with $\mathrm{HO}$ defects. (b) $\Gamma-\mathrm{J}$ PM with $\mathrm{H} 1$ defects. The inset shows the lasing spectrum above the threshold. Circles and triangles denote longer and shorter wavelengths, respectively. 


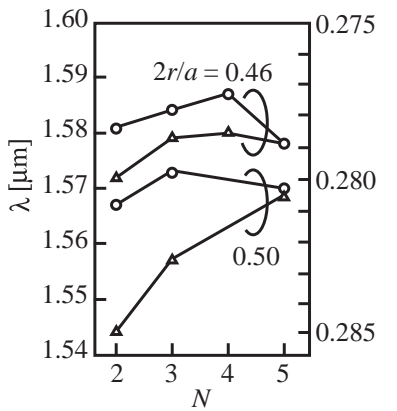

(a)

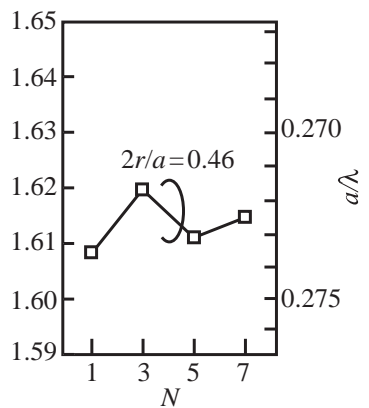

(b)

Fig. 7 Lasing wavelengths $\lambda$ (left axis) and corresponding $a / \lambda$ (right axis) measured as functions of $N$ in PMs with H1 defects. (a) $\Gamma-J$ PM. (b) $\Gamma-\mathrm{X}$ PM. 